\title{
Repercussões do tratamento de câncer de mama sobre a funcionalidade de membro superior
}

\author{
Repercussions of breast cancer treatment on upper limb functionality
}

\author{
Fisioterapia em oncologia mamária \\ Physiotherapy in mammary oncology
}

\author{
${ }^{1}$ Luana Paula Nava \\ ${ }^{2}$ Bibiana Ferrari Barroso \\ ${ }^{3}$ Alexandre Simões Dias \\ ${ }^{4}$ Amanda Sachetti
}

\section{Endereço Científico:}

Rua Capitão Eleutério 610 Edifício Centro Executivo Sala 705/706 - Passo Fundo/RS CEP 99070190

CEP: 99070190 - (54) 99154-3394

amandasachetti@gmail.com
Resumo

Introdução: as terapêuticas utilizadas para o controle do câncer de mama envolvem cirurgia, tratamentos sistêmicos e radioterapia, com isso, um aglomerado de complicações pode impactar a funcionalidade do membro superior.

Objetivo: avaliar a força muscular e a amplitude de movimento de mulheres com câncer de mama em tratamento radioterápico e analisar a influência destas variáveis na funcionalidade do membro homolateral a cirurgia.

Métodos: assim, foram incluídas 57 mulheres e realizada avaliação da amplitude de movimento e força muscular comparando membro superior homolateral e contralateral a cirurgia e, após aplicado o questionário de desabilidades do ombro, braço e mão.

Resultados: os resultados mostraram escore satisfatório de funcionalidade do membro superior. A força muscular mostrou-se inalterada, porém houve redução na amplitude de movimento em flexão e abdução. Foi verificada correlação entre amplitude de movimento, força muscular e funcionalidade principalmente em abdução.

Conclusão: a força muscular afeta diretamente a funcionalidade do membro superior.

Descritores: Neoplasias da mama. Radioterapia. Complicações pós-operatórias. Incapacidade funcional. Extremidade superior.

\begin{abstract}
Introduction: Therapies for breast control involve surgery, systemic treatments and radiotherapy, so a cluster of complications can impact the functionality of the superior.

Objective: To evaluate the muscular strength and range of motion of women with breast cancer in radiotherapy treatment and to analyze an influence of these variables on the functionality of the member homolateral to the surgery. Methodology: Thus, we included 57 women and appreciation assessment of range of motion and muscle strength comparing upper limb homolateral and contralateral to surgery and, after applied in the Disabilities of the arm, shoulder and hand questionnaire.

Results: The results showed satisfactory superior functionality score. A muscle strength was unchanged, but there was a reduction in the range of motion in flexion and abduction. Correlation between range of motion, muscle strength and functionality was verified mainly in abduction. Conclusion: Muscle strength directly affects the functionality of the upper limb.
\end{abstract}

Keywords: Breast Neoplasms. Radiotherapy. Postoperative complications. Functional incapacity. Upper extremity.

\footnotetext{
Cite como

Nava LP, Barroso BF, Dias AS, Sachetti A. Repercussões do tratamento de câncer de mama sobre a funcionalidade de membro superior. Conscientiae Saúde 2019 jul./set.; 18(3):402-413. https://doi.org/10.5585/ConsSaude.v18n311016.
} 


\section{Introdução}

O câncer de mama $(\mathrm{CM})$ conceitua-se como o resultado de uma multiplicação desordenada de células de diversas formas clínicas e morfológicas ${ }^{1}$. Apresenta-se por ser a doença maligna mais comumente encontrada e consequentemente a maior causa de óbito na população feminina brasileira ${ }^{2}$. De acordo com dados do Instituto Nacional do Câncer, foram estimados 57.960 casos novos no Brasil em 2016, resultando em um grave problema de saúde pública devido às crescentes estatísticas ${ }^{3}$.

Apesar do desenvolvimento das técnicas cirúrgicas, ainda há uma tendência ao diagnóstico tardio, repercutindo na escolha dos tratamentos e nas complicações provenientes destes. A cirurgia impacta significativamente na funcionalidade e, quando associada à linfadenectomia axilar (LA) aumenta a morbidade do ombro homolateral ao procedimento. Dentre as principais complicações pós-tratamento destacam-se a redução da força muscular (FM), rigidez articular e limitação da amplitude de movimentos (ADM) ${ }^{4}$.

Tem-se a quimioterapia, a radioterapia e a hormonioterapia como tratamentos complementares e, durante ou após a realização destes, efeitos colaterais e comorbidades podem surgir ou vir a somar aos já existentes. Há um vasto comprometimento funcional do complexo articular do ombro decorrentes do tratamento oncológico, como a dor articular, parestesias, restrição da mobilidade, fadiga e diminuição da FM, prolongando o retorno da paciente as suas atividades cotidianas. Desta maneira, faz-se necessário o acompanhamento fisioterapêutico a fim de prevenir e/ou controlar tais repercussões ${ }^{5,6}$.

Com base no contexto apresentado, o estudo tem por objetivo avaliar a FM e a ADM de mulheres com CM em tratamento radioterápico em um hospital de referência no norte do Rio Grande do Sul e analisar a influência destas variáveis na funcionalidade do membro superior homolateral a cirurgia.

\section{Material e métodos}

Tipo do estudo

Trata-se de um estudo do tipo transversal, descritivo e analítico. Aprovado pelo Comitê de Ética em Pesquisa das instituições envolvidas através do parecer $n^{\circ}$ 2.033.038. Além disso, seguindo os princípios éticos previsto na resolução 466/2012 CNS, as participantes da pesquisa assinaram o Termo de Consentimento Livre e Esclarecido (TCLE). 


\section{População e Amostra}

A população elencada para o presente estudo foi de mulheres com CM. A amostra foi então composta por todas as mulheres, com diagnóstico confirmado de CM através de exame anatomopatológico e que se encontravam em tratamento radioterápico na instituição hospitalar durante os meses de maio a agosto de 2017.

As mesmas necessariamente precisavam ter sido submetidas a procedimento cirúrgico, e não ter ingressado para a reabilitação fisioterapêutica até o momento da coleta de dados. $\mathrm{O}$ tratamento quimioterápico e/ou hormonioterápico não foi um critério utilizado.

Foram excluídas mulheres que não consentiram participar da pesquisa; neoplasia de mama com acometimento bilateral; portadoras de outros tipos de carcinoma; presença de metástases ósseas ou cerebrais; doença neurológica com sequelas; doença reumática agudizada e aquelas que apresentaram estado de confusão mental ou inabilidade para compreender os comandos solicitados pelo fisioterapeuta.

\section{Procedimentos}

Inicialmente foi realizada uma triagem nos prontuários disponíveis no setor de radioterapia, observando o resultado do exame anatomopatológico, confirmando neoplasia de mama e exposto o convite da pesquisa. Após o aceite, um questionário semiestruturado foi aplicado com a finalidade de conhecer o perfil sociodemográfico, história clínica e questões relacionadas aos tratamentos.

O exame físico foi composto pela avaliação da ADM e FM. Para analisar a ADM foram consideradas as técnicas de goniometria segundo Marques ${ }^{7}$, comparando o membro homolateral à cirurgia com o contralateral nos movimentos de flexão, abdução e rotação interna.

Para quantificar a FM, foi utilizada a proposta descrita por Kendall et al. ${ }^{8}$, de forma manual, baseado em cinco graus para classificar o tipo de FM desenvolvida. Sendo que o grau 0 representa nenhuma evidência de contração muscular palpável ou visível e grau 5 caracteriza o movimento através da amplitude completa contra a gravidade e uma resistência máxima. Em ambas as avaliações as participantes permaneceram em bipedestação, executando os movimentos de abdução e flexão, e em decúbito dorsal efetuando a rotação interna de ombro, contra a ação gravitacional. 
E, para a avaliação do desempenho funcional do membro superior foi utilizado o DASH (Disabilities of the Arm, Shoulder and Hand Questionnaire - Questionário de desabilidades do ombro, braço e mão). Trata-se de um questionário validado, contendo 30 questões, com pontuação que varia de 1 (houve pouca dificuldade) à 5 (não conseguiu fazer). Tais questões são designadas para medir as funções físicas e os sintomas. Ainda, existem dois módulos opcionais referentes à performance musical ou esportiva e trabalho, não aplicados nesta amostragem. Indica-se ausência de dificuldade para a atividade quando escore é entre 0 e 25 pontos; pouca dificuldade quando escore é entre 26 e 50 pontos; média dificuldade quando escore é entre 51 e 71 pontos; acima de 76 pontos considera-se dificuldade acentuada e 100 pontos equivale a limitação funcional total ${ }^{9}$.

\section{Análise Estatística}

A base de dados foi construída em uma planilha do Microsoft Excel e a análise estatística foi realizada utilizando-se o IBM SPSS Statistics versão 22 para Windows. As variáveis descritivas referentes à caracterização amostral e terapêutica foram apresentadas por meio de média, desvio padrão e porcentagem. As variáveis numéricas relacionadas à ADM e FM foram expressas como mediana (percentil 25 - percentil 75 ), além da funcionalidade nos diferentes tipos cirúrgicos. E, as associações entre funcionalidade, medida pelo escore DASH e FM ou ADM foram descritas utilizando-se o coeficiente de correlação ordinal de Spearman, que varia de -1 a +1 , considerando 0 uma correlação nula; 1 correlação perfeita; $0,2<r<0,4$ correlação fraca; $0,4<\mathrm{r}<0,7$ correlação moderada; $0,7<\mathrm{r}<0,9$ correlação forte, possuindo o mesmo significado para casos negativos ou positivos ${ }^{10}$.

\section{Resultados}

A amostra inicial foi constituída por 64 mulheres portadoras de CM. Destas, foram excluídas sete participantes, quatro por apresentarem metástases locorregionais variadas, e três pelo comprometimento bilateral decorrente de mastectomia associada à LA, totalizando em uma amostra de 57 pacientes.

A maioria das participantes era casada, com predomínio da raça branca, apresentavam baixa escolaridade e, não estavam expostas aos fatores de risco externos investigados (Tabela 1). 
Tabela 1 - Perfil sociodemográfico e fatores de risco

\begin{tabular}{|l|l|l|l|}
\hline & Média & \multicolumn{1}{|c|}{ DP } & \\
\cline { 2 - 4 } & & & \\
\hline Idade & 54,2 & 11,3 & \\
\hline Estado Civil & & & 17,5 \\
\hline Solteira & & & 57,8 \\
\hline Casada & & & 17,5 \\
\hline Viúva & & 7 \\
\hline Divorciada & & \\
\hline Raça & & & 94,7 \\
\hline Branca & & 3,5 \\
\hline Negra & & & 1,75 \\
\hline Parda & & \\
\hline Escolaridade & & & 66,6 \\
\hline Primeiro Grau & & & 10,5 \\
\hline Segundo Grau & & & 8,7 \\
\hline Terceiro Grau & & & 8,7 \\
\hline Ensino Superior & & 3,5 \\
\hline Pós-Graduação & & & \\
\hline Histórico familiar & & 68,4 \\
\hline Sim & & 31,5 \\
\hline Não & & \\
\hline Tabagismo & & 10,5 \\
\hline Sim & & & 89,4 \\
\hline Não & & & \\
\hline Etilismo & & & \\
\hline Sim & & & 87,7 \\
\hline Não & & \\
\hline & & \\
\hline
\end{tabular}

Valores expressos em média, desvio padrão (DP) e porcentagem $(\%)$

Na tabela 2 estão descritos os procedimentos cirúrgicos mais evidenciados, a realização concomitante da LA, as modalidades terapêuticas assumidas e suas respectivas combinações. 
Tabela 2 - Caracterização do tratamento oncológico

\begin{tabular}{|c|c|c|}
\hline Variável & $\mathbf{N}$ & $\%$ \\
\hline \multicolumn{3}{|l|}{ Tipo de Cirurgias } \\
\hline Setorectomia & 25 & 43,8 \\
\hline Nodulectomia & 9 & 15,7 \\
\hline Quadrantectomia & 8 & 14,0 \\
\hline Mastectomia Parcial & 10 & 17,5 \\
\hline Mastectomia Total & 5 & 8,7 \\
\hline \multicolumn{3}{|l|}{ Linfadenectomia Axilar } \\
\hline Sim & 49 & 85,9 \\
\hline Não & 8 & 14,0 \\
\hline \multicolumn{3}{|c|}{ Associações de Tratamento } \\
\hline Cirurgia + Radioterapia & 3 & 5,2 \\
\hline Cirurgia + Quimioterapia + Radioterapia & 8 & 14,0 \\
\hline Cirurgia + Radioterapia + Hormonioterapia & 15 & 26,3 \\
\hline Cirurgia + Quimioterapia + Radioterapia +Hormonioterapia & 31 & 54,3 \\
\hline
\end{tabular}

Quando aplicado o questionário de avaliação da funcionalidade (DASH) na população estudada, obteve-se uma mediana do escore total de 12,5 (2,5 - 41,6). No entanto, a mediana do escore aumentou nas mulheres submetidas a cirurgias radicais 33,3 (8,7 - 45,8) quando comparado as que realizaram cirurgias conservadoras $8,7(2,5-37,7)$.

Já, quando avaliada a FM e ADM no membro homolateral e contralateral a cirurgia, foi observado manutenção da FM nos movimentos analisados, mas, redução de ADM em flexão e abdução, principalmente (Tabela 3). 
Tabela 3 - Força muscular e amplitude de movimento no membro homolateral e contralateral à cirurgia $(n=57)$

\begin{tabular}{|c|c|}
\hline Variável & Descrição \\
\hline \multicolumn{2}{|l|}{ Membro homolateral } \\
\hline \multicolumn{2}{|l|}{ Força muscular } \\
\hline Flexão & $5,0(3,0-5,0)$ \\
\hline Abdução & $5,0(3,5-5,0)$ \\
\hline Rotação Interna & $5,0(4,0-5,0)$ \\
\hline \multicolumn{2}{|l|}{ Amplitude de movimento } \\
\hline Flexão & $150,0(137,5-170,0)$ \\
\hline Abdução & $141,0(115,0-165,5)$ \\
\hline Rotação Interna & $90,0(90,0-90,0)$ \\
\hline \multicolumn{2}{|l|}{ Membro contralateral } \\
\hline \multicolumn{2}{|l|}{ Força muscular } \\
\hline Flexão & $5,0(5,0-5,0)$ \\
\hline Abdução & $5,0(5,0-5,0)$ \\
\hline Rotação Interna & $5,0(5,0-5,0)$ \\
\hline \multicolumn{2}{|l|}{ Amplitude de movimento } \\
\hline Flexão & $161,0(150,0-180,0)$ \\
\hline Abdução & $160,0(140,0-180,0)$ \\
\hline Rotação Interna & $90,0(90,0-90,0)$ \\
\hline
\end{tabular}

A tabela 4 apresenta os dados referentes à correlação das variáveis ADM e FM com funcionalidade. Foi identificada maior correlação entre FM e funcionalidade, principalmente no movimento de abdução do ombro. 
Tabela 4 - Correlações entre funcionalidade, força muscular e amplitude de movimento no membro homolateral à cirurgia $(\mathrm{n}=57)$

\begin{tabular}{|l|l|}
\hline \multicolumn{1}{|c|}{ Variável } & \multicolumn{1}{c|}{$\begin{array}{c}\text { Correlação com } \\
\text { Funcionalidade }\end{array}$} \\
\hline Força muscular & \\
\hline Flexão & $-0,73$ \\
\hline Abdução & $-0,76$ \\
\hline Rotação Interna & $-0,71$ \\
\hline Amplitude de movimento & \\
\hline Flexão & $-0,49$ \\
\hline Abdução & $-0,68$ \\
\hline Rotação Interna & $-0,30$ \\
\hline
\end{tabular}

Valores expressam coeficiente de correlação ordinal de Spearman.

Sinal (-) significa que as variáveis correlacionadas variam em sentido contrário.

\section{Discussão}

Este estudo incluiu mulheres com CM tratadas em uma instituição de referência em oncologia no norte gaúcho.

A média de idade encontrada nesta amostra foi em torno dos 50 anos, considerada uma população adulta não idosa, assemelhando-se aos dados da Internacional Agency for Research on Câncer $^{11}$, que revelou uma estimativa de 2.589.299 novos casos de neoplasia mamária para o ano de 2035, sendo que 1.538 .973 serão diagnosticados em mulheres com idade inferior aos 65 anos.

A predominância da raça branca, a baixa escolaridade, e o estado civil evidenciado neste estudo, convergem com uma recente literatura que analisou o perfil clínico, sociodemográfico e epidemiológico de 299 mulheres com $\mathrm{CM}^{12}$. Além disso, outros pesquisadores reforçam os achados referentes às tais características elucidadas ${ }^{13}$.

Nesta pesquisa, a maioria das participantes não era tabagista ativa e não consumia álcool. No entanto, um estudo caso-controle, acompanhou 4402 mulheres com CM onde foram identificados os subtipos da neoplasia e a exposição ao fator de risco, e concluíram que o consumo de álcool associa-se ao desenvolvimento do subtipo luminal A. Já, o hábito tabágico ( $\geq 20$ cigarros/dia) possui tendências significativas para desenvolver o subtipo luminal A, B, e 
HER2-negativo ${ }^{14}$. Porém, sabe-se que existem outros fatores ambientais ou genéticos envolvidos no desenvolvimento de neoplasias. Em pesquisa desenvolvida por Gaudet et al. ${ }^{15} \mathrm{o}$ histórico familiar, principalmente o parentesco de primeiro grau estava positivamente associado ao surgimento de todos subtipos moleculares de CM. No presente estudo, a susceptibilidade genética foi um fator de risco amplamente visualizado.

Nesta investigação, houve predominância das cirurgias conservadoras sobre as radicais, corroborando com um perfil clínico-patológico indiano, que indicou uma prevalência de $63 \%$ de cirurgias conservadoras na população em evidência ${ }^{16}$. Em contrapartida, uma literatura brasileira mostrou predomínio de mulheres mastectomizadas, devido ao diagnóstico em estágios avançados e dimensões tumorais importantes ${ }^{4}$. Diante disso, Veiga et al. ${ }^{17}$ concluiu que há piores resultados na capacidade funcional de mastectomizadas, reforçando os achados deste estudo, uma vez comparada a funcionalidade do membro superior no pós-operatório de cirurgias radicais versus conservadoras.

A cirurgia somada ao tratamento radioterápico, quimioterápico e hormonioterápico constatado em maior porcentagem amostral, vai ao encontro de outras pesquisas, que resultaram em dados similares referente às combinações terapêuticas ${ }^{12,18}$. A literatura relata inúmeras disfunções físicas relacionadas às terapias do $\mathrm{CM}$, entre elas alterações de funcionalidade ${ }^{6}$.

Apesar das participantes serem expostas a vários tratamentos antineoplásicos, não foi observado déficit significativo de funcionalidade do membro homolateral a cirurgia assemelhando-se a dois estudos respectivamente. O primeiro avaliou 105 mulheres em pósoperatório tardio, e verificou que as pacientes retomaram suas atividades de vida diária sem apresentarem dificuldades ${ }^{5}$. E, o segundo, composto por 45 mulheres observou que a ADM de flexão, abdução e rotações do membro homolateral comparado ao colateral não apresentou diferenças expressivas, não prejudicando o desempenho funcional do membro superior ${ }^{19}$.

As principais limitações de movimento encontradas neste estudo foram em flexão, mas especialmente em abdução do ombro, confirmando com a literatura ${ }^{20}$, que evidenciou déficit nestes mesmos movimentos, com ênfase no comprometimento em abdução. Em uma pesquisa semelhante, foram analisadas 28 mulheres no período pré-operatório, pós-operatório imediato e tardio, e constatou-se diminuição significativa na ADM de flexão e abdução de ambos os membros superiores quando comparados à mensuração pré-operatória. Quando comparado o período pré-operatório com o pós-tardio, verificou-se diferença apenas no lado homolateral a cirurgia $^{21}$. 
Foi constatado que houve associação entre ADM e a capacidade funcional do membro superior, semelhante ao que foi encontrado por Thomas-Maclean et al. ${ }^{22}$, que avaliou 347 mulheres, e destas $59 \%$ manifestaram restrição de movimento influenciando consideravelmente na capacidade em desenvolver suas atividades. Em outra pesquisa, foram analisadas 741 pacientes no período de 6-12 meses pós-cirurgia, onde foram realizadas cinco avaliações incluindo ADM e aplicação do DASH, com intervalo de um ano entre elas. Os resultados mostraram importante associação entre ADM e DASH, além de que, a abdução de ombro apresentou-se significativamente mais restrita na primeira avaliação do que nas subsequentes ${ }^{23}$.

Como visualizado, a FM e a funcionalidade exibiram forte correlação, pelo fato da força estar inalterada, influenciando positivamente na função do membro superior. Moraes et al. ${ }^{24}$ salientam os benefícios que o treinamento de força pode proporcionar as mulheres que enfrentam o CM. Espera-se com a prática dos exercícios mudanças imediatas na ADM do ombro, aumento da densidade óssea, progressão da FM e equilíbrio.

Os resultados encontrados ratificam a real importância e necessidade da abordagem fisioterapêutica em todos os níveis do tratamento para o $\mathrm{CM}$, sendo esta uma das principais condutas preventivas de complicações.

Como limitação, considera-se a amostra heterogênea e não ter sido analisado o tempo de pós-operatório. Contudo, a amostra é representativa da realidade e mostrou aspectos importantes relacionados ao impacto da FM e ADM na função física destas mulheres.

\section{Conclusão}

Observa-se com esta pesquisa que as pacientes não apresentaram grau insatisfatório referente à capacidade funcional do membro homolateral a cirurgia, sendo que este resultado pode ocorrer devido à adaptação das mulheres a nova condição ou as poucas limitações decorrentes do procedimento cirúrgico.

A ADM e a FM principalmente, afeta diretamente a funcionalidade do membro superior. Em consequência disso, preza-se pelo início do acompanhamento fisioterapêutico na fase préoperatória, para que as sequelas pós-operatórias sejam minimizadas e assim, a reabilitação seja facilmente conduzida e potencializada. Sugerem-se novas pesquisas com ênfase em FM e funcionalidade de membro superior, com subsequente elaboração de protocolos de exercícios de fortalecimento para mulheres em tratamento de CM. 


\section{Referências}

1. Quinto SMG, Mejia DPM. Benefícios da fisioterapia no tratamento de linfedema pósmastectomia radical: uma revisão literária [Monografia]. Goiânia(GO): Faculdade Ávila; 2012.

2. Rett MT, Santos AKG, Mendonça ACR, Oliveira IA, DeSantana JM. Efeito da fisioterapia no desempenho funcional do membro superior no pós-operatório de câncer de mama. Rev Ciência \& Saúde. 2013;6(1):18-24.

3.Instituto Nacional de Câncer (INCA). [homepage na internet; 2015]. Estimativa 2016| Incidência de Câncer no Brasil [acesso em 20 de agosto 2017]. Disponível em: http://www.inca.gov.br/estimativa/2016/estimativa-2016-v11.pdf.

4. Assis MR, Marx AG, Magna LA, Ferrigno ISV. Late morbidity in upper limb function and quality of life in women after breast cancer surgery. Braz J Phys Ther. 2013;17(3):236-46.

5. Sousa E, Carvalho FN, Bergmann A, Fabro EAN, Dias RA, Koifman RJ. Funcionalidade de membro superior em mulheres submetidas ao tratamento do câncer de mama. Revista Brasileira de Cancerologia. 2013;59(3):409-17.

6. Hayes SC, Johansson K, Stout NL, Prosnitz R, Armer JM, Gabram SI et al. Upper-Body Morbidity After Breast Cancer. Wiley Online Library. 2012;118(8):2237-49.

7. Marques AP. Manual de goniometria: ângulos articulares dos membros superiores. $2^{\mathrm{a}}$ ed. São Paulo: Manole; 2003.

8. Kendall F, Mccreary E, Provance P. Provas de função muscular. $4^{\mathrm{a}}$ ed. São Paulo: Manole; 1995

9. Orfale AG, Araújo PMP, Ferraz MB, Natour J. Translation into brazilian portuguese, cultural adaptation and evalution of the disabilities of the arm, shoulder and hand questionnaire. Braz $\mathbf{J}$ Med Biol Res. 2005;38(2): 293-302.

10. Dancey CP, Reidy J. Estatística sem matemática para psicologia: usando SPSS para windows. $5^{\text {a }}$ ed. Porto Alegre: Artmed; 2013.

11. Internacional Agency for Research on Cancer. Estimated number of new cancers. [acesso em 26 de novembro de 2017]. Disponível em: http://globocan.iarc.fr/Pages/burden_sel.aspx.

12. Magalhães G, Souza CB, Fustitoni SM, Matos JC, Schirmer J. Perfil clínico, sociodemográfico e epidemiológico da mulher com câncer de mama. J.res.:fundam.care.online. 2017;9(2):473-79.

13. Rechia TL, Prim AC, Luz CM. Upper limb functionality and quality of life in women with five-year survival after breast cancer surgery. Rev Bras Ginecol Obstret. 2017;39(3):115-21. 
14. Ellingjord-Dale M, Vos L, Hjerkind KV, Hjartaker A, Russnes HG. Alcohol, physical activity, smoking and breast cancer subtypes in a large nested case-control study from the Norwegian Breast Cancer Screening Program. Cancer Epidemiol Biomarkers Prev. 2017:1-23.

15. Gaudet MM, Gierach G, Carter B, Luo J, Milne R, Weiderpass E et al. Risk factors by molecular subtypes of breast cancer: a pooled analysis of nine cohorts. American Association for Cancer Research. 2017;77(13):1-5.

16. Wadasadawala T, Nair N, Lewis A, Budrukkar A, Parmar V, Gupta S et al. Clinical profile and outcome of various stages of breast cancer treated at a Tertiary Cancer Centre. Clinical Oncology. 2017;29(3):72-83.

17. Veiga DF, Campos FSM, Ribeiro LM, Junior IA, Filho JV, Juliano Y et al. Mastectomy versus conservative surgical treatment: the impact on the quality of life of women with breast cancer. Rev. Bras. Saúde Matern. Infant. 2010;10(1):51-7.

18. Winters-Stone KM, Dobek J, Bennett JA, Nail LM, Leo MC, Schawartz A. The effect of resistance training on muscle strength and physical function in older, postmenopausal breast cancer survivors: a randomized controlled trial. J Cancer Surviv. 2012;6(2):189-99.

19. Velloso FSB, Barra AA, Dias RC. Functional performance of upper limb and quality of life after sentinel lymph node biopsy of breast cancer. Rev Bras Fisioter. 2011;15(2):146-153.

20. Gois MC, Furtado PR, Ribeiro SO, Lisboa LL, Viana ESR, Micussi MTABC. Amplitude de movimento e medida de independência functional em pacientes mastectomizadas com linfadenectomia axilar. Rev. Ciênc. Med. 2012;21(6):111-18.

21. Bregagnol RK, Dias AS. Alterações funcionais em mulheres submetidas a cirurgia de mama com linfadenectomia axilar total. Revista Brasileira de Cancerologia. 2010;56(1):25-33.

22. Thomas-Maclean RL, Hack TF, Kwan WB, Towers A, Miedema B, Tilley A. Arm morbidity and disability after breast cancer: new directions for care. Oncol Nurs Forum. 2008;35(1):65-71.

23. Boquiren VM, Hack TF, Roanne L, Towers A, Kwan WB, Tilley A. A longitudinal analysis of chronic arm morbidity following breast cancer surgery. Breast Cancer Research and Treatment. 2016;157(3):413-25.

24. Moraes A, Battaglini C, Schimidt LF, Rafael AD, Roussenq SC, Dias M et al. Treinamento de força e câncer de mama: uma revisão sistemática. Revista Brasileira de Prescrição e Fisiologia do Exercício. 2014;9(49):664-73. 